\section{Headache in children is associated with obesity}

Obesity is associated with headache in adults, but the relationship between weight and headache has not previously been studied thoroughly in children and adolescents. Hershey et al. have now shown that BMI is positively correlated with headache frequency and headacheassociated disability in children and that weight loss reduces headache frequency in pediatric patients who are overweight.

This retrospective multicenter study supported by the American Headache Society examined 913 consecutive patients (mean age $11.9 \pm 3.4$ years). Headache frequency and headache-related functional disability were recorded at index visit. Children were classed as overweight if their BMI was above the $85^{\text {th }}$ percentile of the Centers for Disease Control standard values or obese if their BMI was at or above the $95^{\text {th }}$ percentile. A total of 213 $(23.3 \%)$ patients were followed up at 3 months and $174(19.1 \%)$ at 6 months.

The prevalence of obesity in this headache population was not significantly different to that in the general pediatric population (17.5\% vs $17.1 \%)$. There was a positive correlation between BMI and both headache frequency and functional disability score $(r=0.10$, $P=0.003$ and $r=0.08, P=0.02$, respectively). In overweight or obese children, a reduction in $\mathrm{BMI}$ at follow-up significantly correlated with a reduction in headache frequency $(r=0.32$; $P=0.01$ at 3 months and $r=0.34 ; P=0.01$ at 6 months).

The authors suggest that obesity does not cause headache, but rather exacerbates existing headache problems. Weight reduction might prove to be an important aspect of headache treatment in children who are overweight.

Original article Hershey AD et al. (2008) Obesity in the pediatric headache population: a multicenter study. Headache [doi:10.1111/j.1526-4610.2008.01232.x]

\section{Right-to-left shunt has no impact on recurrent stroke risk}

A new study has reported that the presence of a right-to-left shunt (RLSh) resulting from patent foramen ovale, regardless of its magnitude, does not seem to be a risk factor for recurrent stroke in patients with cryptogenic stroke. Previous studies have found that RLSh is associated with a first ischemic stroke, but few have assessed the contribution of this risk factor in stroke recurrence.

Serena and colleagues prospectively studied 486 patients with cryptogenic stroke (388 ischemic stroke, 98 transient ischemic attack) at 17 hospitals in Spain. Contrast transcranial Doppler imaging was used to detect the presence and magnitude of RLSh in patients while they performed the Valsalva maneuver. Transthoracic and/or transesophageal echocardiography, CT scan or MRI of the brain was also performed.

RLSh was observed in 297 (61.1\%) patients, $200(41.2 \%)$ of whom displayed a massive RLSh. Younger patients ( $<55$ years old) were more likely than the cohort as a whole to exhibit a RLSh $(70.7 \%$ vs $52.5 \%)$ in general or a massive RLSh (51.5\% vs $31.9 \%)$. Over a mean follow-up of $729.2 \pm 410.8$ days, stroke recurrence occured in 28 patients. Logisticregression analysis revealed no association between massive RLSh and recurrent stroke in either the younger group or the whole cohort. Treatment type (anticoagulation or antiplatelet) did not affect the rate of stroke recurrence.

The authors discourage the use of potentially aggressive treatments, such as anticoagulation therapy, for the prevention of recurrence in patients with massive RLS and suggest that patent foramen ovale occlusion should be performed only within the framework of current clinical trials.

Original article Serena J et al. (2008) Recurrent stroke and massive right-to-left shunt: results from the prospective Spanish multicenter (CODICIA) study. Stroke 71: 888-895 